\title{
Development of Attention Networks and Their Interactions in Childhood
}

\author{
Joan P. Pozuelos \\ University of Granada
}

\author{
Alejandro Castillo and Luis J. Fuentes \\ University of Murcia
}

\author{
Pedro M. Paz-Alonso
enter on Cognition, Brain, and Language, \\ Pedro M. Paz-Alonso
Basque Center on Cognition, Brain, and Language, \\ Donostia, Spain
}

M. Rosario Rueda

University of Granada

\begin{abstract}
In the present study, we investigated developmental trajectories of alerting, orienting, and executive attention networks and their interactions over childhood. Two cross-sectional experiments were conducted with different samples of 6- to 12-year-old children using modified versions of the attention network task (ANT). In Experiment $1(N=106)$, alerting and orienting cues were independently manipulated, thus allowing examination of interactions between these 2 networks, as well as between them and the executive attention network. In Experiment $2(N=159)$, additional changes were made to the task in order to foster exogenous orienting cues. Results from both studies consistently revealed separate developmental trajectories for each attention network. Children younger than 7 years exhibited stronger benefits from having an alerting auditory signal prior to the target presentation. Developmental changes in orienting were mostly observed on response accuracy between middle and late childhood, whereas executive attention showed increases in efficiency between 7 years and older ages, and further improvements in late childhood. Of importance, across both experiments, significant interactions between alerting and orienting, as well as between each of these and the executive attention network, were observed. Alerting cues led to speeding shifts of attention and enhancing orienting processes. Also, both alerting and orienting cues modulated the magnitude of the flanker interference effect. These findings inform current theoretical models of human attention and its development, characterizing for the first time, the age-related course of attention networks interactions that, present in adults, stem from further refinements over childhood.
\end{abstract}

Keywords: attention networks, child attention network task, development, network interactions

Functions of attention include achieving and maintaining a state of alertness, orienting toward and selecting sensory events for preferred processing, and regulating thoughts and responses in a goal-directed effortful mode. This variety of functions has been associated with distinct brain networks (Petersen \& Posner, 2012). The alerting network involves the locus coeruleus and areas of the

This article was published Online First July 28, 2014.

Joan P. Pozuelos, Department of Experimental Psychology, Center for Research on Mind, Brain, and Behavior (CIMCYC), University of Granada; Pedro M. Paz-Alonso, Basque Center on Cognition, Brain, and Language, Donostia, Spain; Alejandro Castillo and Luis J. Fuentes, Department of Basic Psychology and Methodology, University of Murcia; M. Rosario Rueda, Department of Experimental Psychology, CIMCYC, University of Granada.

Research presented in this article was supported by grants from the Spanish Ministry of Science and Innovation to M. Rosario Rueda (PSI2011.27746) and Luis J. Fuentes (CSD2008-00048 and PSI201123340), as well as an FPI Fellowship (BES-2009-017932) awarded to Joan P. Pozuelos.

Correspondence concerning this article should be addressed to M. Rosario Rueda, Department of Experimental Psychology, Center for Research on Mind, Brain, and Behavior (CIMCYC), Campus Cartuja s/n, University of Granada, Granada 18071, Spain. E-mail:rorueda@ugr.es frontal and parietal cortices. The orienting network includes subcortical structures such as the superior culliculus, as well as the superior parietal cortex, temporoparietal junction, and frontal eye fields. Finally, the executive attention network comprises the anterior cingulate cortex, lateral and ventral prefrontal cortex, and the basal ganglia. Following this neurocognitive model, Fan, McCandliss, Sommer, Ras, and Posner (2002) developed the attention network task (ANT), an experimental task that provides a measure of the efficiency of each of these three networks. In the current research, we aimed at adapting the ANT to study the development of attention networks during childhood, as well as the possible interactions between networks along this period.

Both the ability to sustain attention (tonic alertness) and the ability to increase response readiness evoked by external cues (phasic alertness) are functions of the alerting network. Developmental studies have shown differences between children and adults in both speed of preparation from alerting cues and maintenance of that preparation (Morrison, 1982). These phasic and tonic aspects of alertness influence reaction times (RTs) when alertness is measured by comparing trials with and without warning cues. Just as adults, young children reduce their RTs to stimuli that are preceded by warning cues (Berger, Jones, Rothbart \& Posner 2000). However, despite the fact that children and adults encode warning signals at equivalent rates, children appear to be less efficient (i.e., 
have longer RTs) processing the warning aspect of the cue (Kraut, 1976; Smothergill \& Kraut, 1989).

Regarding mechanisms of the orienting network, there appears to be little differences between children and adults in the ability to shift attention to exogenous cues. However, age-related improvements are documented for the speed of moving attention voluntarily (Akhtar \& Enns, 1989; Brodeur \& Enns, 1997), and the ability to disengage attention from a location or object (Enns, 1990; Trick \& Enns, 1998). Studies involving shifting attention toward peripheral cues that indicate the location of a target stimulus show no changes between 6-year-old children and adults (Rueda et al., 2004). In contrast, studies that require re-orientation of attention, as when cues are presented opposite to the location of the subsequent target, have shown that the time to disengage from the location of the cue is reduced with age (Akhtar \& Enns, 1989). When the orienting effect is computed by comparing RTs to validly versus invalidly cued targets, a sharp development of orienting is observed until about late childhood (Schul, Townsend, \& Stiles, 2003; Waszak, Li, \& Hommel, 2010). The original ANT triggers orienting by means of valid exogenous cues, but it does not provide information about the processes of disengagement and re-orienting of attention because invalid orienting cues are not presented.

Executive attention is often studied by tasks that involve conflict between different dimensions of a target stimulus, as in the Stroop task, or between the target and distracting information, as in the Flanker task (Posner, Rueda, \& Kanske, 2007). Developmental studies about executive control of attention have suggested that this network strongly develops during early childhood (Rueda, 2014; Rueda, Posner, \& Rothbart, 2005). Specifically, children typically progress from an almost complete inability to carry out conflict tasks to a relatively good performance on these tasks between ages 2 and 7 years (Gerardi-Caulton, 2000; Rueda et al., 2005). A study using a flanker task with arrows pointing left or right showed that children between the ages of 7 and 9 years experience greater interference than adults from incongruent flanking arrows, particularly at the response selection level (Ridderinkhof \& van der Molen, 1995). Additional data with other tasks involving executive attention indicate that this function shows a protracted development during childhood (Kray, Eber, \& Linderberger, 2004) and depending on the demands of executive processes (e.g., working memory) may extend to adolescence and early adulthood (Davidson, Amso, Cruess, \& Diamond, 2006; Diamond, 2013). A recent study using a color version of the flanker task reported a steady development of conflict processing during childhood and adolescence and up to adulthood, as well as a decline in older adults (Waszak et al., 2010).

Most prior studies examining the development of attention networks have been carried out with tasks that measure each network independently. One advantage of the ANT is that it provides a measure of the three attention networks within the same experimental procedure, thus allowing examination of interactions between them. Rueda et al. (2004) developed a child-friendly version of the ANT, wherein the arrows of the original ANT were replaced with colorful fish. The child ANT was used to conduct a series of studies with children ages $6-10$ years and young adults intended to trace the developmental course of the attention networks (Rueda et al., 2004). In that study, no changes in the orienting effect with age were observed. However, results showed stability of the alerting effect during middle childhood, but a significant reduction of the alerting score between 10 -year-olds and adults. Likewise, a decrease in the size of the flanker interference effect was found between ages 6 and 7, and little or no difference from 7 years up to adulthood.

\section{Interactions Between Attention Networks}

Although the attention networks are associated with distinct brain circuits, there is evidence suggesting that they are not functionally independent (Callejas, Lupiañez, \& Tudela, 2004). Fan et al.'s (2002) study showed a significant interaction between cue and flanker conditions, showing increased flanker interference effects under cues conveying alertness. To further study interactions between the attention networks, Callejas et al. (2004) developed a modified version of the ANT. In their task, different trial events were used to manipulate alerting (auditory tone) and orienting (valid and invalid visual cues). Their results revealed significant interactions between alerting and orienting, as well as between each of these networks and the executive attention network. The Alerting $\times$ Orienting interaction indicated that the orienting effect was larger in conditions with alerting cues. Evidence from subsequent studies suggests that alerting could both speed shifts of attention (Callejas, Lupiáñez, Funes, \& Tudela, 2005) and enhance selection with cue-target intervals of up to $500 \mathrm{~ms}$ (Fuentes \& Campoy, 2008). Additionally, both alerting and orienting cues modulated the size of the flanker interference effect. Alerting cues lead to larger flanker interference, an effect that has been interpreted as indicative of an inhibitory relation between the alerting and executive attention networks. The idea is that fast and automatic responses are prioritized over controlled behavior after preparation cues are presented (Posner, 1994). However, data from a recent study with adults suggest that alerting may also broaden the attentional focus leading to increased processing of distracting information in the flanker task (Weinbach \& Henik, 2012). On the other hand, greater interference effect in trials with invalid orienting cues compared with valid or no-cue trials has also been reported (Callejas et al., 2005). This indicates that correct orientation of attention prior to the occurrence of the target may help focusing attention and filtering out distracting stimulation, whereas the opposite occurs in trials with invalid cues, allowing more interference from distracters.

To our knowledge, no prior study has examined interactions among the attention networks in detail during childhood. The present study was conducted with the intention of filling that lack. By assessing interactions between attention functions, we hoped to help explain inconsistent data about the developmental course of each function. Given that alerting and orienting functions appear to have an earlier developmental course than that of executive attention, we expected to observe modulation of these networks over executive control from early childhood. Also, we expected that this modulatory effect would have greater impact when less efficient forms of executive control are likely to be observed, as in early compared with middle or late childhood. At early ages, the executive system may largely benefit from conditions that facilitate filtering out irrelevant information. With this purpose, we modified the child ANT according to changes introduced by Callejas et al. (2004) and conducted two studies with 6- to 12-year-old children. 


\section{Experiment 1}

\section{Method}

Participants. Participants in Experiment 1 were recruited from local schools in an urban area of southern Spain. Children $(N=106)$ ranging in age from 6 to 12 years participated in the study. Informed consent was obtained from caregivers prior to participation. Children with normal or corrected-to-normal vision and no history of developmental disorders were included in the study. Descriptive statistics of the sample are presented in Table 1. There was no significant differences in total intelligence score, as measured with the Kaufman Brief Intelligence Test (K-BIT; Kaufman \& Kaufman, 1990) between age groups, $F(1,97)=1.47, p>$ .10 .

Task and stimuli. A schematic representation of the experimental task is presented in Figure 1. Each trial started with either an alerting cue consisting on a $50 \mathrm{~ms}-$ long $2,000-\mathrm{Hz}$ tone presented in $50 \%$ of trials or a blank frame for the same duration with no tone. Four hundred milliseconds after the alerting cue, a visual orienting cue (i.e., an asterisk) was presented on two thirds of the trials for $100 \mathrm{~ms}$. No cue was presented in the remaining one third of trials. The orienting cue was presented about $1^{\circ}$ of visual angle above fixation on half of the trials and about $1^{\circ}$ below it on the remaining half. Also, on half of the trials, the cue was presented in the same location as the upcoming target (i.e., valid cue), whereas in the other half it was presented in the opposite location (i.e., invalid cue). Fifty milliseconds after the orienting cue, the target array was presented either above (in half of the trials) or below fixation. The target array consisted of a row of five yellow fish, which remained on the screen until a response was made or up to $2,500 \mathrm{~ms}$. In half of the trials, the fish in the middle pointed to the same direction as those on the sides (congruent trials), whereas it pointed to the opposite direction as the flanking fish (incongruent trials) in the remaining half. Each fish subtended $1.6^{\circ}$ of visual angle, and the contours of adjacent fish were separated by $0.21^{\circ}$ viewed at an approximated distance of $50 \mathrm{~cm}$. Animated feedback lasting $1 \mathrm{~s}$ was presented after completion of the target frame. The feedback consisted of the middle fish blowing bubbles and moving the tail together with the sound "Woohoo!" for correct responses or a single tone and no animation for incorrect or omitted responses. A fixation cross displayed at the center of the screen was presented throughout the trial and remained on the screen during the intertrial interval, which had a random duration of between 500 and 4,200 ms. After completion of each experimental block, a between-block feedback was presented, providing information on both accuracy and speed of response. The presentation of the stimuli and collection of responses was controlled with E-Prime (Version 2; Psychology Software Tools).

Procedure. The experimental sessions were conducted in small groups of six children at once in a quiet classroom at school. Children wore headphones while performing the task. Each session consisted of one practice block of 24 trials and six experimental blocks of 48 trials each, a total of 288 experimental trials, 24 trials of each of the 12 experimental conditions: 2 (alerting: tone vs. no-tone) $\times 3$ (orienting: valid-cue, invalid-cue, no-cue) $\times 2$ (executive attention: congruent vs. incongruent). Conditions were randomly chosen in each trial. Accuracy and speed of responses were registered in each trial.

Participants were told that a group of five fish would appear on the screen, either above or below the fixation cross. They were instructed to pay attention to the fish in the center of the array and feed it by pressing the button of the mouse that matched the direction in which the fish was pointing. An instruction block consisting on four trials (two congruent and two incongruent) where conducted to explain the task. Finally, participants were encouraged to maintain fixation at the central cross throughout the task and to respond as quickly and accurately as possible.

\section{Results}

All participants had a percentage of errors that did not exceed 2 standard deviations from the mean of its particular age group, and therefore none was excluded from the study. The percentage of trials with errors of omission was low $(M=1.51, S D=1.56)$ and did not differ $(F<1)$ among age groups, therefore accuracy analysis only takes into consideration the percentage of commission errors.

Attention networks scores. Scores for each attention network were computed using both RTs and percentage of errors. The alerting score was computed by subtracting median RTs or percentage of errors in trials with tone from trials without a tone. The orienting score was calculated by subtracting the dependent measures on trials with the valid cue from those with the invalid cue. Finally, the executive attention score was the result of subtracting median RTs or percentage of errors in trials with congruent flankers from trials with incongruent flankers. The left side of Table 2 presents the attention network scores obtained by each age group

Table 1

Descriptive Data of Participants in Experiments 1 and 2: Means (and Standard Deviations) of Age in Months and Composite IQ for Each Age Group

\begin{tabular}{|c|c|c|c|c|c|c|}
\hline \multirow[b]{2}{*}{ Age group } & \multicolumn{3}{|c|}{ Experiment 1} & \multicolumn{3}{|c|}{ Experiment 2} \\
\hline & $n$ (males) & Age in months & IQ score & $n$ (males) & Age in months & IQ score \\
\hline 6 years & $11(4)$ & $80(2.9)$ & $108(9.7)$ & $13(7)$ & $80(1.8)$ & $113(6.8)$ \\
\hline 7 years & $12(8)$ & $90(3.6)$ & $108(6.9)$ & $22(12)$ & $89(3.0)$ & 110 (10.9) \\
\hline 8 years & $28(11)$ & $97(2.8)$ & $108(11.3)$ & $28(19)$ & $101(3.7)$ & $106(13.1)$ \\
\hline 9 years & $17(9)$ & $112(3.3)$ & $103(9.2)$ & $21(12)$ & $112(2.7)$ & $106(10.2)$ \\
\hline 10 years & $13(5)$ & 127 (3.4) & $100(10.2)$ & $22(13)$ & $124(3.4)$ & 105 (8.9) \\
\hline 11 years & $14(9)$ & $136(3.2)$ & $104(7.8)$ & $26(14)$ & $137(3.0)$ & $108(8.1)$ \\
\hline 12 years & $11(5)$ & $149(5.0)$ & $105(15.0)$ & $18(9)$ & $146(2.1)$ & 109 (10.8) \\
\hline
\end{tabular}




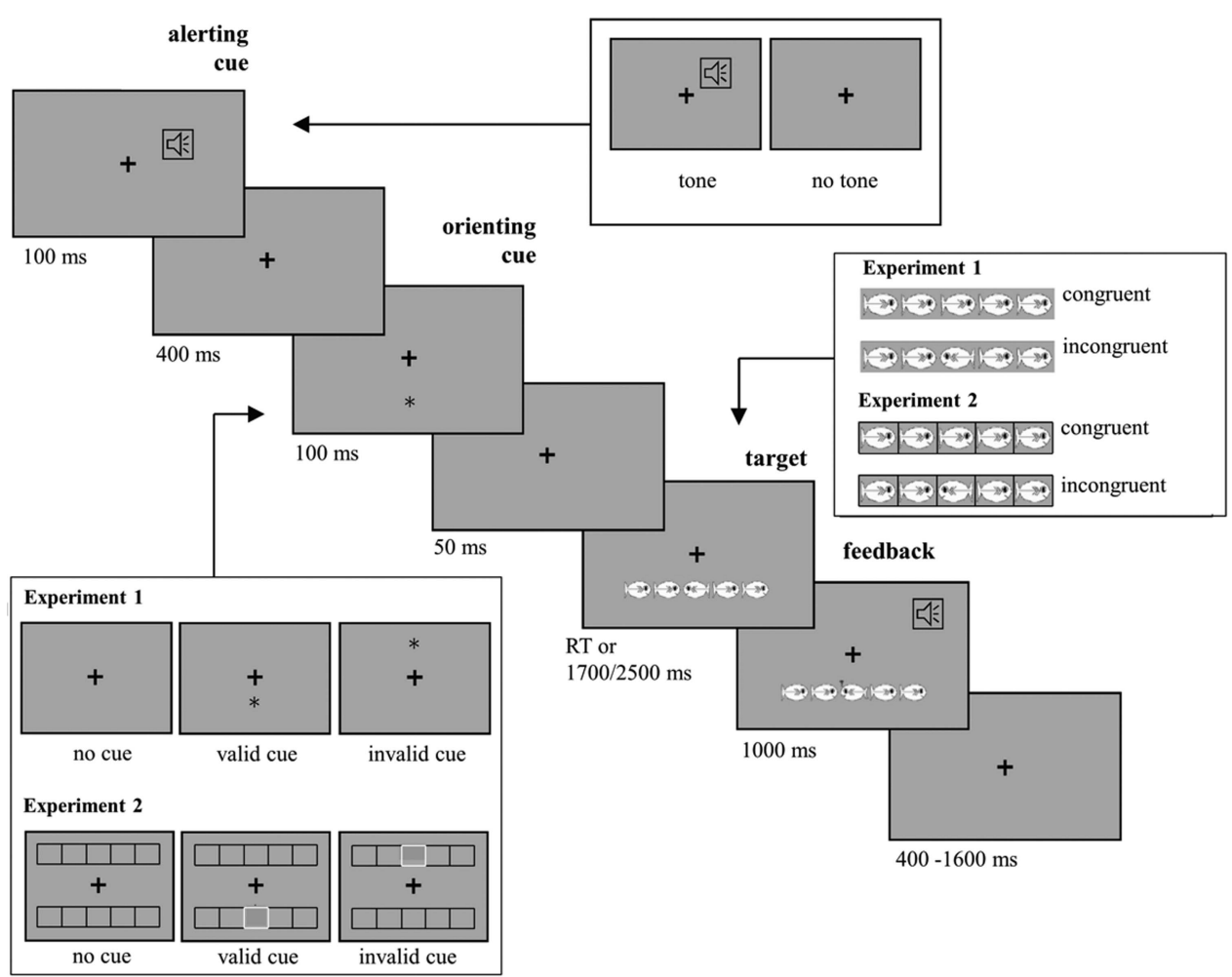

Figure 1. Schema of the experimental tasks used in the study. In Experiment 2, the location of stimuli was signaled by squares that were present throughout the task. $\mathrm{RT}=$ reaction time.

in Experiment 1. Group differences on these scores were analyzed by means of one-way analyses of variance (ANOVAs) with age as the between-subjects factor. The main effect of age was significant for the alerting score, $F(6,99)=3.52 ; p<.01$, and the executive attention score, $F(6,99)=2.61, p<.05$, but did not reach statistical significance for the orienting score, $F(6,99)=1.94, p=$ .08. To further explore age differences in the network scores, we first performed Fisher's least significant difference tests in order to determine where differences between groups emerged. Then, we clustered age groups that did not differ from each other to carry out planned comparisons. For ease of communication, only data from planned comparisons are reported. Those analyses revealed a significant reduction in the alerting score between the ages of 6-7 years and 8-12 years, $F(1,99)=19.21, p<.01$. As for the executive attention score, 6- to 8-year-olds differed significantly from 9- to 12 -year-olds, $F(1,99)=11.64 ; p<.001$. Also, the oldest group differed significantly from 6- to 8-year-olds, $F(1$, $99)=11.9 ; p<.001$, but only marginally from 9- to 11-year-olds, $F(1,99)=3.40 ; p=.07$. Regarding the orienting network score, we conducted planned contrasts to assess differences between the youngest children and the rest of the groups. Comparisons revealed significant differences for the contrasts 6 versus $7, F(1,99)=$

Table 2

Attention Networks Scores With Reaction Times (in Milliseconds) and Response Accuracy (Percentage of Errors) Per Age Group Obtained in Experiments 1 and 2

\begin{tabular}{|c|c|c|c|c|c|c|c|c|c|c|c|c|}
\hline \multirow[b]{3}{*}{ Age group } & \multicolumn{6}{|c|}{ Experiment 1} & \multicolumn{6}{|c|}{ Experiment 2} \\
\hline & \multicolumn{2}{|c|}{ Alerting score } & \multicolumn{2}{|c|}{ Orienting score } & \multicolumn{2}{|c|}{$\begin{array}{l}\text { Executive attention } \\
\text { score }\end{array}$} & \multicolumn{2}{|c|}{ Alerting score } & \multicolumn{2}{|c|}{ Orienting score } & \multicolumn{2}{|c|}{$\begin{array}{l}\text { Executive attention } \\
\text { score }\end{array}$} \\
\hline & RT & $\%$ errors & RT & $\%$ errors & RT & $\%$ errors & RT & $\%$ errors & RT & $\%$ errors & RT & $\%$ errors \\
\hline 6 years & 30 (19.7) & $0.6(3.8)$ & $85(33.5)$ & 9.3 (11.5) & $70(33.2)$ & $5.5(9.5)$ & $28(31.0)$ & $0.6(5.0)$ & $95(29.2)$ & $6.2(4.5)$ & $51(25.7)$ & $6.4(6.1)$ \\
\hline 7 years & $32(13.2)$ & $1.7(4.9)$ & 64 (27.3) & $6.0(6.3)$ & $78(28.3)$ & $7.7(6.5)$ & 31 (19.6) & $2.2(4.4)$ & 87 (27.3) & $5.1(5.4)$ & $59(32.3)$ & $6.9(5.1)$ \\
\hline 8 years & $17(18.3)$ & $-0.4(2.8)$ & 77 (23.9) & $3.2(3.5)$ & 65 (28.2) & $3.6(4.6)$ & $22(23.0)$ & $1.7(3.9)$ & 88 (29.6) & $3.5(4.3)$ & $56(21.5)$ & $4.2(3.1)$ \\
\hline 9 years & 10 (18.9) & $0.2(2.1)$ & $72(23.1)$ & $4.3(4.5)$ & $54(22.4)$ & $5.3(4.6)$ & $24(18.5)$ & $1.7(2.8)$ & $90(30.5)$ & $2.5(3.4)$ & $49(21.1)$ & $4.3(3.2)$ \\
\hline 10 years & $14(17.7)$ & $0.5(1.9)$ & $58(16.8)$ & $3.6(8.8)$ & $60(23.9)$ & $6.2(10.9)$ & $16(16.0)$ & $0.7(3.6)$ & $81(19.2)$ & $1.6(3.3)$ & $39(23.4)$ & $3.7(2.5)$ \\
\hline 11 years & $12(15.1)$ & $-0.02(1.5)$ & 79 (23.6) & $2.1(3.0)$ & 58 (10.4) & $3.9(2.7)$ & $16(16.8)$ & $0.8(2.3)$ & 87 (21.5) & $1.6(2.4)$ & $41(22.5)$ & $2.0(2.1)$ \\
\hline 12 years & $8(19.1)$ & $0.06(2.1)$ & $64(25.9)$ & $2.2(2.4)$ & $42(16.0)$ & $2.8(2.6)$ & $10(19.2)$ & $0.6(1.2)$ & $82(16.8)$ & $1.9(2.2)$ & $45(19.0)$ & $2.0(1.6)$ \\
\hline
\end{tabular}

Note. Standard deviations are in parentheses. $\mathrm{RT}=$ reaction time. 
$3.95, p<.05$; 6 versus $10, F(1,99)=7.02, p<.01$; and 6 versus $12, F(1,99)=3.83, p=.05$, years. Regarding analyses with errors, the main effect of age was significant only for the orienting score, $F(6,99)=2,16 ; p<.05$. Subsequent planned comparisons revealed a significant reduction in the orienting score between 6-7 and $8-12$ years, $F(1,99)=57.08, p<.001$. There was no significant effect of age for either the alerting or the executive attention scores $(F \mathrm{~s}<1)$.

Interactions among attention networks. Data summarizing interactions among networks in this experiment are presented in the leftmost part of Table 3. To examine the interaction between alerting and orienting networks, we computed orienting scores separately for each alerting condition and entered these scores in a 2 (alerting) $\times 7$ (age) mixed-model ANOVA. Likewise, executive scores were computed separately for each of the alerting (tone vs. no tone) and orienting (valid vs. invalid) conditions to examine Alerting $\times$ Executive and Orienting $\times$ Executive interactions and their possible modulation by age. Results revealed a significant modulation of orienting conditions over executive scores with both RTs, $F(1,99)=94.61, p<.001$, and percentage of errors, $F(1$, $99)=94.61, p<.001$, but no modulation of alerting over either orienting or executive attention scores (all $F \mathrm{~s}<1$ ). Moreover, age qualified none of these effects.

\section{Discussion}

As predicted, results of Experiment 1 revealed separate developmental trajectories for each attention network, which was evinced by changes in the network scores as a function of age. With RTs, the alerting score was significantly reduced after age 7 , with no further changes from age 8 to 12 years. With errors, the alerting score is expected to be negative, as it has been largely documented in adults, who tend to commit more errors when a warning cue is provided than when no warning cues precede the target (Posner, 1978). In the age range here studied, the alerting score was close to zero for children 8 to 12 years, but greater than zero for the youngest children (i.e., 6-7 years). Scores

Table 3

Interactions Among Networks

\begin{tabular}{|c|c|c|c|c|}
\hline \multirow[b]{2}{*}{ Interaction } & \multicolumn{2}{|c|}{ Experiment 1} & \multicolumn{2}{|c|}{ Experiment 2} \\
\hline & RT & $\%$ errors & RT & $\%$ errors \\
\hline (a) Alerting $\times$ Orienting & $n s$ & $n s$ & * & $n s$ \\
\hline No tone & $70(31)$ & $3.0(4.9)$ & $83(35)$ & $2.8(4.6)$ \\
\hline Tone & $72(29)$ & $3.5(5.5)$ & $91(31)$ & $2.5(4.2)$ \\
\hline \multicolumn{5}{|l|}{ (b) Alerting $\times$ Executive } \\
\hline Attention & $n s$ & $n s$ & $n s$ & $* * *$ \\
\hline No tone & $43(41)$ & $3.3(6.8)$ & $39(46)$ & $4.4(6.8)$ \\
\hline Tone & $48(47)$ & $4.1(6.7)$ & $41(41)$ & $2.0(5.9)$ \\
\hline $\begin{array}{l}\text { (c) Orienting } \times \text { Executive } \\
\text { Attention }\end{array}$ & **** & **** & *** & **** \\
\hline Valid & $44(31)$ & $1.9(4.6)$ & $32(25)$ & $2.3(3.5)$ \\
\hline Invalid & $89(35)$ & $6.3(7.2)$ & $75(34)$ & $5.1(5.9)$ \\
\hline
\end{tabular}

Note. Orienting scores with both reaction times (RTs) and percentage of errors are presented in function of alerting conditions (a). Executive scores with RT and percentage of error are shown for particular alerting (b) and orienting (c) conditions. Data in bold indicate that the interaction is qualified by age (see Figure 2). $n s=$ nonsignificant $(p>1$ ).

${ }^{*} p<.05 .{ }^{* * * *} p<.001$ greater than zero indicate that more errors are committed in the no-tone than the tone condition. If young children have poorer tonic alerting levels, as RT data suggest, having a warning cue prior to the target is likely to produce beneficial rather than harmful effects on response accuracy because the cue can help on regaining attention on the task.

The effect of age on the orienting network score was more clear-cut for errors than for RTs. Nevertheless, in both measures, the youngest group showed larger scores than older children, meaning that their responses were comparatively slower and less accurate when having to reorient attention to the location of the target. Many studies examining exogenous orienting effects with children and adults have used orienting cues consisting of luminance changes of a frame that defines the area in which the target stimulus would be located (Ortega, López, Carrasco, Anllo-Vento, \& Aboitiz, 2013; Schul et al., 2003; Waszak et al., 2010), as in the original orienting paradigm proposed by Posner (1980). The nature of the cues used in the current study may account for the differences observed in our study and those finding significant reductions in the RT-orienting effect along childhood. This hypothesis was tested in the next experiment.

Regarding the executive attention score, differences were observed between young children, ages 6-8 years and 9-12 years, and a further decrease trend was observed for the oldest group. In the study by Rueda et al. (2004) in which the original child ANT was used, no further decreases in the executive score were observed after age 7 ; however, the inclusion of invalid orienting cues in the current study is likely to have made the task more difficult, leading to steeper developmental changes in this network. In fact, a significant Orienting $\times$ Executive Attention interaction was observed in our study, indicating that the executive score was larger under invalid orienting conditions, as will be discussed later.

Regarding interactions among networks, we only found a significant Orienting $\times$ Executive Attention interaction with both RTs and errors. This interaction was due to a reduction of the flanker interference effect under valid orienting cues compared with invalid cues (see Table 3). Children of all ages showed equivalent rates of benefit from valid cues over focalization of attention and suppression of irrelevant stimuli. This can be related to the fact that we did not find a clear effect of age on the RT orienting score in our study. To test whether the nature of the cue was responsible for the lack of age differences in the orienting effect, we conducted a second experiment in which frames were used to enclose the location of stimuli. Altering the luminance of the frames would serve to foster exogenous orienting by cues.

\section{Experiment 2}

\section{Method}

Participants. A total of 159 children ranging in age between 6 and 12 years, who met the same inclusion criteria as in Experiment 1 and whose parents/tutors provided informed consent, participated in Experiment 2. There was no significant differences in total intelligence score between age groups, $F(1,149)=1.59$, $p>$.10. Data from children with more than 2 standard deviations above the mean percentage of errors of their age group $(n=9)$ were discarded from the analyses. Descriptive data of the final sample are presented in the rightmost part of Table 1. 
Task, stimuli, and procedure. In Experiment 2, the location of stimuli was marked with squares that were presented throughout the entire task. The orienting cue consisted of highlighting the position of the square in the middle, either above or below the fixation point. The square could be highlighted either in the same location as the target (valid cue) or in the opposite location (invalid cue). In the no-cue condition, the square was not highlighted (see Figure 1). The remaining stimuli and timing parameters were identical to the ones used in Experiment 1 as it was the procedure followed to administer the task.

\section{Results}

The overall percentage of omission errors was $2.07(S D=2.44)$, and no differences were observed between age groups $(F<1)$ in this measure.

Attention networks scores. The rightmost part of Table 2 presents the scores for each attention network and age group with both RT and errors. One-way ANOVAs with RTs showed a statistically significant main effect of age for the alerting, $F(6$, $143)=2.64, p<.05$, and executive attention, $F(6,143)=2.21$, $p<.05$, scores, but not for orienting scores $(F<1)$. The developmental course of alerting was assessed with planned comparisons, which revealed significant differences between the 6- to 9-year olds and the 10- to 12-year-olds, $F(1,143)=12.79 ; p<$ .001 , given that alerting scores of the 8 - to 9 -year-olds differed significantly from those of the 10 - to 12 -year-olds, $F(1,143)=$ $5.16, p<.05$, but not from those of the 6- to 7-year-olds, $F(1$, $143)=2.22, p=.14$. Regarding the executive attention score, a statistically significant reduction was observed between the ages of $6-9$ and $10-12$ years, $F(1,143)=9.27, p<.01$.

Regarding errors, we found a statistically significant effect of age for the orienting, $F(6,143)=4.17, p<.001$, and executive attention, $F(6,143)=5.68, p<.001$, scores. No effect of age was obtained for the alerting score $(F<1)$. The orienting score followed a linear reduction between age 6 and age 10, and no changes were seen between age 10 and age $12, F(1,143)=22.83$, $p<.001$. On the other hand, the curve of the executive attention score showed two inflection points, one between 6-7 years and $8-10$ years, $F(1,143)=11.54, \mathrm{p}<001$, and a second one between $8-10$ and $11-12$ years, $F(1,143)=8.72, p<.01$.

Interactions among networks. The rightmost part of Table 3 presents data summarizing interactions among networks in Experiment 2. Analyses of interactions were conducted following the same procedure as in Experiment 1. Alerting conditions modulated the size of orienting scores as calculated with RTs, $F(1,142)=$ $4.48, p<.05$. Likewise, orienting conditions modulated the size of executive scores as calculated with both RTs, $F(1,142)=192.04$, $p<.001$, and errors, $F(1,142)=27.69, p<.001$. Finally, alerting conditions modulated executive scores only when calculated with errors, $F(1,142)=18.69, p<.001$. However, this last effect was qualified by Age, $F(6,142)=5.16, p<.001$. The Alerting $\times$ Age interaction with executive scores as dependent variable is presented in Figure 2. Post hoc contrasts were performed using Bonferroni-adjusted alpha levels. Results revealed that the flanker interference effect was significantly larger in the no-tone condition than in the tone condition for 6-year-old, $F(1,142)=19.37, p<$ .001 , and 7-year-old $F(1,142)=21.08, p<.001$, children, whereas it did not reach statistical significance for 8-year-old, $F(1$,

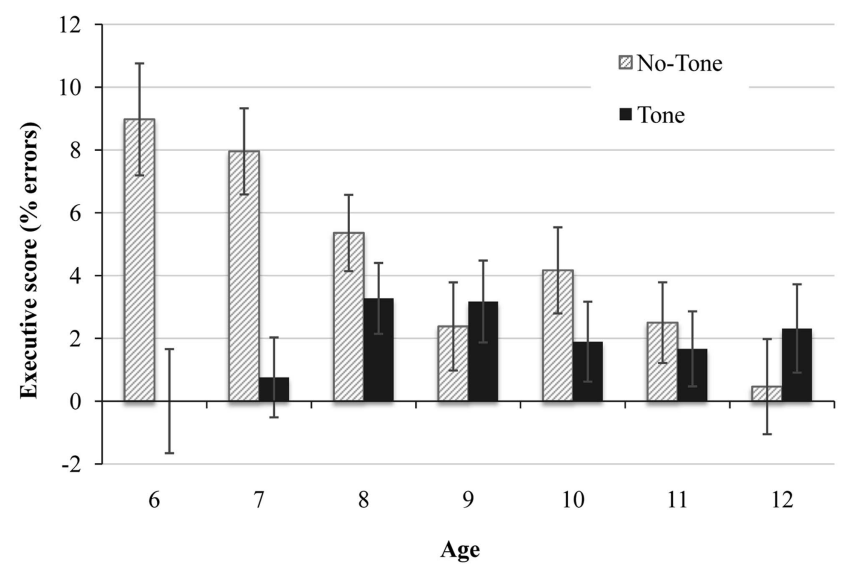

Figure 2. Graph depicts executive attention scores in function of alerting conditions and age group obtained in Experiment 2. Age is given in years. Vertical bars represent standard errors of the means.

$142)=2.25, p=.14,9$-year-old, $F<1,10$-year-old, $F(1,142)=$ $2.10, p=.15,11$-year-old, $F<1$, and 12-year-old, $F(1,142)=$ $1.14, p=.29$, children.

\section{Comparison of Experiments 1 and 2}

To test for effects due to changes introduced in Experiment 2 with respect to Experiment 1 and to examine whether the developmental trajectories of the attention networks were influenced by those changes, we carried out a series of factorial ANOVAs in which experiment $(1,2)$ and age $(6,7,8,9,10,11$, and 12 years) were included as between-participants factors and each of the network scores as dependent measures. In these ANOVAs, the Experiment $\times$ Age interaction was not statistically significant for any network score using either RT or accuracy measures (all $F$ s $<$ 1). However, we found a significant main effect of Experiment in RT analyses for both the orienting, $F(1,242)=21.7, p<.001$, and the executive attention, $F(1,242)=14.7, p<.001$, scores. This result suggests that the experimental changes introduced in Experiment 2 produced larger orienting effects $(72 \mathrm{~ms}$ in Experiment1 vs. $87 \mathrm{~ms}$ in Experiment 2) and reduced executive attention scores (61 ms in Experiment 1 vs. $49 \mathrm{~ms}$ in Experiment 2). The effect of age was significant with alerting, $F(6,242)=5.22, p<$ .001 , and executive attention, $F(6,242)=3.99, p<.001$ scores, but only marginal, $F(6,242)=1.99, p=.07$, with orienting scores. Age changes in alerting were found between 6- to 7-yearolds and the older groups, $F(1,242)=26.1, p<.001$. Also, a linear reduction between ages 8 and 12 years reached statistical significance, $F(1,242)=5.94, p<.05$. Regarding executive attention, the developmental curve showed an inflection point between ages 8 and 9 years (significant groups comparison in $6-8$ years vs. 9-12 years) $F(1,242)=20.11, p<.001$.

Analyses using networks scores with percentage of comission errors revealed marginal main effects of experiment with alerting, $F(1,242)=3.61, p=.06$, and orienting scores, $F(1,242)=3.47$, $p=.06$. These effects were due to a trend for higher alerting error percentage scores in Experiment $2(M=1.2, S D=0.27)$ compared to Experiment $1(M=0.4, S D=0.32)$, as well as lower orienting scores in Experiment $2(M=3.2, S D=0.40)$ relative to 
Experiment $1(M=4.4, S D=0.48)$. The effect of age was significant with orienting, $F(6,242)=5.36, p<.001$, and executive attention, $F(6,242)=3.72, p<.01$, scores, but not with alerting scores $(F<1)$. Orienting scores were significantly larger for 6- to 7-year-olds than for the rest of the age groups, $F(1$, $242)=28.75, p<.001$. Regarding executive attention, differences were observed between $6-7$ years and $8-10$ years, $F(1,242)=$ $6.77, p<.01$, and also between children $8-10$ and $11-12$ years old, $F(1,242)=5.97, p<.05$.

Figure 3 presents the developmental trajectories of each attention network by plotting scores for both RT (Figure 3a) and response accuracy (Figure $3 b$ ) as a function of age with data from Experiments 1 and 2 combined.

\section{Discussion}

Framing the location of stimuli causes a general reduction in executive attention scores by facilitating focusing of attention on the central target, hence, diminishing the processing of distracting flankers. Additionally, orienting cues utilized in Experiment 2 appear to produce stronger attention capture than those used in Experiment 1, leading to greater difficulties to disengage attention from the cued location. This is indicated by the fact that RT increases in the orienting score in Experiment 2 with respect to Experiment 1 were only observed in the invalid cue condition, from 653 in Experiment 1 to $680 \mathrm{~ms}$ in Experiment 2, $F(1,242)=$ $13.14, p<.001$, compared with a nonsignificant increase from 580 in Experiment 1 to $593 \mathrm{~ms}$ in Experiment 2 for valid-cue trials. On the other hand, variations introduced to the task did not affect alerting scores. This was expected given that the alerting cue used in the current experiment was identical to the one utilized in the previous study. More interesting is the fact that changes in the experimental conditions did not alter the developmental course of the attention networks, as indicated by the lack of Experiment $\times$ Age interactions.

The interactions among the networks showed a similar pattern in both experiments (see Table 3). However, in Experiment 2, alerting conditions modulated the size of executive scores as calculated with percentage of comission errors, and this modulation changed with age. Young children showed larger executive scores when no alerting cues were presented, whereas from 8 years up children showed equivalent executive scores with and without alerting tone (see Figure 2). Only 12-year-old children showed the pattern usually found in adults (i.e., larger interference scores under higher alerting conditions) although the difference did not reach statistical significance. We believe this result can be explained by young children's poorer level of sustained attention or tonic alertness in the absence of warning cues. This being the case, warning cues should help rather than hinder task performance in young children.

\section{General Discussion}

In the studies presented here, we had two main goals. First, we sought to further understand the development of attention networks over childhood. Second, based on the interactions among the attention networks that have been documented in adults (Callejas et al., 2005; Weinbach \& Henik, 2012), we wanted to examine whether those interactions were present or modulated by age during childhood. According to these goals, a number of modifications were introduced to the original child-version of the ANT (Rueda et al., 2004). We separated alerting and orienting events, which allowed for the independent manipulation of these two networks. We also introduced invalid orienting cues, which provided a measure of orienting that was influenced by processes of disengagement and re-orienting of attention from wrongly cued locations. Additionally, the display of the task was changed in the second experiment, in which the location of stimuli, both target and distractors, was marked by frames, and orienting cues were conveyed by briefly changing the brightness of the central frame.

\section{Development of the Attention Networks}

The developmental curve of the alerting network in RT data showed a significant decline between the younger (i.e., 6 and 7 years) and the older groups, and a progressive reduction from 8 to 12 years of age. Larger alerting scores indicate that young children benefit more than older children from having a warning auditory signal prior to the presentation of the target. This is due to their slower responses when no auditory tone is presented, which is indicative of young children's greater difficulties in maintaining an optimal level of tonic alertness in absence of warning cues. On the other hand, judging by the lack of age effects on the alerting scores
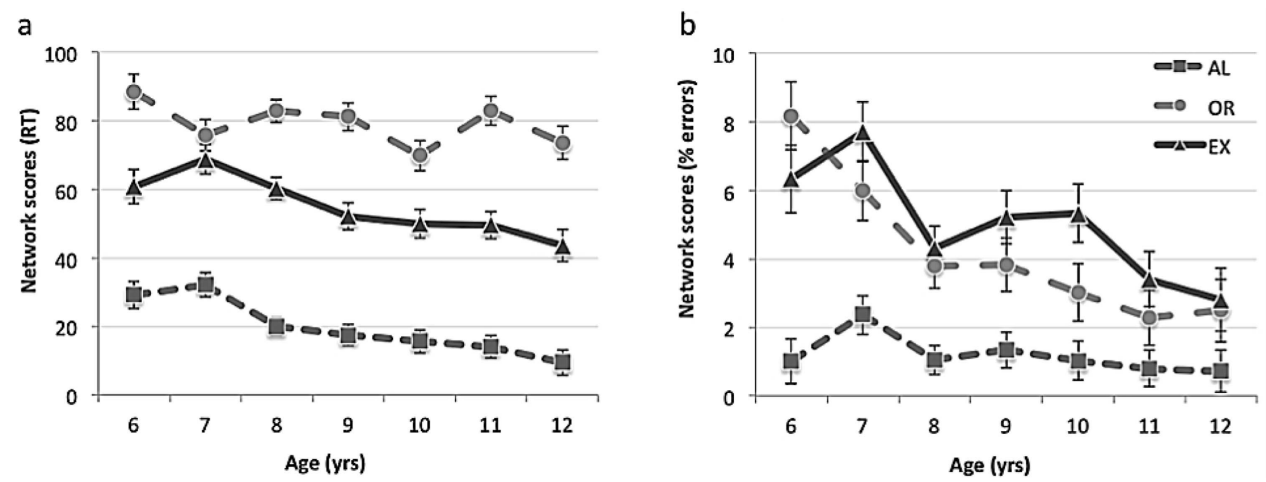

Figure 3. Developmental course of attention networks with reaction time (RT) (a) and percentage of errors (b). Data from Experiments 1 and 2 are combined. Vertical bars represent standard errors of the means. AL = alerting; OR = orienting; $\mathrm{EX}=$ executive attention; $\mathrm{yrs}=$ years. 
calculated with percentage of errors (see Figure 3b), it could be thought that alerting conditions do not affect overall response accuracy across ages. However, the Alerting $\times$ Executive Attention $\times$ Age interaction depicted in Figure 2 indicates that while alerting signals help conflict resolution in young children, they have a harmful effect on conflict resolution in the older groups. These effects of alerting over executive attention suggest that compared with older children, young children show reduced sustained (tonic) alertness, a requisite for the optimal functioning of the attention system.

Young children showed larger orienting scores than the rest of the age groups (see Figure $3 \mathrm{~b}$ ). This indicates that invalid cues compromise response accuracy to a larger extent in children younger than age 8 . Also, 6-year-old children showed larger orienting scores in RTs than children ages 8 and 12 years, suggesting a developmental improvement in orienting and reallocation of attention during the age range studied here. Previous studies have reported a lack of age effects in orienting to valid cues when comparing children beyond age 5 and adults (Enns \& Brodeur, 1989; Wainwright \& Bryson, 2002). In fact, a prior developmental study using the original child ANT, in which only valid orienting cues were used, showed no age-related changes in the orienting score between children of 6 and 10 years of age (Rueda et al., 2004). Modifying the ANT to include invalid orienting cues and calculating the score by subtracting valid-cue trials from invalidcue trials provides a measure of orientation that mainly grasps processes related to disengagement and reallocation of attention. In consonance with our data, significant improvements in children's performance between ages 8 and 11 years have been reported when children have to disengage attention from the attended channel and reallocate it to the other channel in the auditory modality (Pearson \& Lane, 1991) as well as in the visual modality (Waszak et al., 2010). Mounting evidence indicates that switching attention is associated with activation of cortical structures of the parietal and frontal lobes (e.g., Corbetta \& Shulman, 2002). Invalid exogenous cues have been shown to modulate the amplitude of the P300, an event-related potential observed over temporoparietal sites that is associated with activation of the temporoparietal junction (TPJ; Bledowski, Prvulovic, Goebel, Zanella, \& Linden, 2004), a structure within the orienting network. Activation of the TPJ appears to reflect a relatively slow operation of readjustment of attention needed when the expectation of a target at the cued location is broken. Compared with adults, children younger than 8 years of age appear to have a larger modulation of the P300 in response to exogenous invalid cues (Abundis-Gutiérrez, Checa, Castellanos, \& Rueda, 2014). Thus, our results are consistent with the extant empirical evidence and suggest that the function of the orienting network continues developing from middle to late childhood.

Executive attention scores showed a progressive decline with age, which was more pronounced with response accuracy (see Figure 3). With RTs, the developmental curve showed an inflection point between 8 and 9 years of age, whereas with accuracy, differences were observed between $6-7$ and $8-10$ years and also between $8-10$ and 11-12 years. Overall, these data demonstrate that executive attention improves significantly between middle and late childhood. With a simpler version of the ANT (e.g., when only valid orienting cues are used; Rueda et al., 2004), executive attention was shown to have an earlier maturational course. Inclu- sion of invalid orienting cues in one third of the trials caused increased interference from flankers, as indicated by the significant Orienting $\times$ Executive Attention interaction obtained in both experiments (see Table 3). This change in the task led to a more protracted development of conflict resolution. This result is consistent with evidence from previous developmental studies showing age-related reductions of flanker interference beyond age 7 (Fjell et al., 2012; Waszack et al., 2010). In these studies, different versions of the flanker task (e.g., with arrows or geometrical shapes) were used, which could tentatively be more challenging than the fish version utilized in the child ANT.

Much evidence has pointed to the dorsal part of the anterior cingulate cortex and, more generally, the medial frontal cortex, as the main neural node of the brain network involved in cognitive control (Bush, Luu, \& Posner, 2000; Posner, Rothbart, Sheese, \& Tang, 2007; Ridderinkhof, Ullsperger, Crone, \& Nieuwenhuis, 2004). Recently, it has been shown that the extension of the surface area of the medial frontal cortex predicts children's performance of incongruent trials in the flanker task (Fjell et al., 2012). Other structures of the control network are relatively distant areas of the superior and inferior parietal lobe and the precuneus, as well as areas of the inferior frontal cortex and the anterior insula (Dosenbach, Fair, Cohen, Schlaggar, \& Petersen, 2008). Developmental studies conducted with neuroimaging techniques suggest that the frontoparietal network involved in cognitive control shows increased functional connectivity over development, particularly between 7 and 9 years of age and early adolescence (Fair et al. 2007). This is consistent with immature frontoparietal activation shown by 8 - to 12 -year-old children compared with adults while performing the ANT (Konrad et al., 2005). Immature frontoparietal connectivity and activation may be causing a delay in stimulus-to-response translation processes in young children with respect to older children and adults when incongruent information is present in the display (Ridderinkhof, van der Molen, Band, \& Bashore, 1997). This would explain why young children exhibit greater difficulty in reducing interference, as it was observed in the present experiments and in other studies using conflict tasks (Davidson et al., 2006).

\section{Interactions Between the Attention Networks}

The second goal of the present research was to examine whether the interactions between the attention networks observed in adults are present in children and whether they change with age during childhood. A larger orienting effect under conditions of higher alertness was observed in Experiment 2 (see Table 3). This result has been previously reported in adults and interpreted as either an effect of speeding up orientation (Callejas et al., 2005) or enhancing attentional selection (Fuentes \& Campoy, 2008) with increased alertness. Our results are consistent with the idea of alerting improving the function of orienting, either by orienting faster, increasing the selection of the target, or both. Moreover, the fact that no second-order interaction with age was observed suggests either that the influence of alerting over orienting is an essential characteristic of the attention system or that the influence of one network over the other develops earlier than the age range tested in our study. 
Regarding the influence of the alerting and orienting networks over the efficiency of executive attention, two main results were obtained. First, we observed a robust facilitatory effect of orienting over conflict processing, as shown by the reduced flanker effect (i.e., the executive attention score) for both RTs and percentage of errors after a valid orienting cue was provided (see Table 3). Data show that invalid orienting cues lead to much larger RTs and higher error rates in trials with incongruent flankers. Exogenous orienting cues trigger automatic shifts of attention toward the location of the cue (Posner, 1980). Focusing of attention ahead of time helps in filtering out distracting information when the subsequent target appears in that same location. On the contrary, when the target appears in a different location, a potentially effortful process of disengagement and reorienting of attention has to be voluntarily initiated, which leaves less resources available to suppress distracting stimulation.

The second influence on executive attention efficiency was that of alertness. High alerting states are known to cause poorer performance in detection as well as conflict resolution tasks (Aston-Jones, Rajkowski, \& Cohen, 1999; Posner, 1978). This usually results in larger flanker effects following warning cues compared with when no cue is presented. As shown in Table 3, none of our experiments showed that the alerting cues modulated the size of the flanker effect with RT. This lack of interaction indicates that warning cues do not reduce the efficiency of conflict resolution in children. However, in Experiment 2, children showed larger interference effect in response accuracy when no warning cues were presented compared with in trials with auditory tones. More interestingly, this interaction was modified by age (see Figure 2), indicating that while young children (i.e., ages 6-8 years) showed larger interference effects in the absence of warning cues, older children showed either equivalent interference effects in the two alerting conditions or the adult-like pattern showed by the oldest group. This result might be related to young children's difficulties in sustaining attention in absence of alerting cues. It is well documented that that the relationship between alertness and performance is best described by an inverted U-shaped curve, a phenomenon largely known as the Yerkes-Dodson law (Yerkes $\&$ Dodson, 1908). Some authors have suggested that the alertness level of the organism closely follows the tonic level of activation of the locus coeruleus (LC; Aston-Jones \& Cohen, 2005). This model assumes that task performance is best under moderate levels of LC tonic activation but is hampered by either low levels as well as high levels of tonic activation. If young children have low basal levels of tonic alertness, receiving a warning tone prior to having to respond to a target may help them to reach the optimal (i.e., moderate) level of alertness associated with best performance, which in this case is indicated by lower flanker interference scores. In contrast, warning cues have a harmful effect on task performance when subjects already show an adequate level of alertness in the absence of warning signals. In this case, the alerting tone leads to worse conflict processing due to either faster but poorly contrasted responses (Posner, 1994) or enhanced processing of flankers (Weinbach \& Henik, 2012). Thus, the relationship between basal tonic alertness and level of performance may underlie the observed interaction between the alerting and executive attention networks in children.

\section{Concluding Remarks}

The ANT has been widely used to study the function of the attention networks since it was first introduced (Fan et al., 2002). A child version of the task was published 2 years later as an approach to studying the typical development of attention (Rueda et al., 2004). This task has been extensively used to examine attention function in developmental disorders (Houwink, Aarts, Geurts, \& Steenbergen, 2011; Johnson et al., 2008; Kratz et al., 2011) and to examine the effect of environmental (Mezzacappa, 2004), educational (Rueda, Checa, \& Cómbita, 2012; Tao, Marzecova, Taft, Asanowicz, \& Wodniecka, 2011; Yang, Yang, \& Lust, 2011), and other behavioral and neurological (Abdullaev, Posner, Nunnally, \& Dishion, 2010; Dye, Baril, \& Bavelier, 2007; Quintero-Gallego, Gomez, Morales, \& Marquez, 2011) conditions on the efficiency of attention. Despite of the steady increase in the use of the ANT, only a few studies have been carried out to characterize the developmental trajectories of the attention networks in typically developing children, and no prior studies have examined interactions between the attention networks in childhood.

Our results provide further information on the development of the three attention functions during childhood. Alerting showed a significant developmental improvement after age 7 , mostly in speed of processing. Executive attention showed increases in efficiency between 7 years and older ages, and further improvements in late childhood. In turn, benefits of orienting on response precision seem to improve considerably between early and late childhood. Further, 6- to 12-year-old children also showed interactions between alerting and orienting, and orienting and executive attention, which are observed in adults. This suggests that these interactions build up from mechanisms that are inherent to the way the attention networks are connected with each other in the human brain. On the other hand, the effect of the state of alertness on the accuracy of conflict processing appears to evolve according to children gains on the capacity to endogenously sustain alertness with age.

\section{References}

Abdullaev, Y., Posner, M. I., Nunnally, R., \& Dishion, T. J. (2010). Functional MRI evidence for inefficient attentional control in adolescent chronic cannabis abuse. Behavioural Brain Research, 215, 45-57. doi: 10.1016/j.bbr.2010.06.023

Abundis-Gutiérrez, A., Checa, P., Castellanos, C., \& Rueda, M. R. (2014). Electrophysiological correlates of attention networks in childhood and early adulthood. Neuropsychologia, 57, 78-92. doi:10.1016/j .neuropsychologia.2014.02.013

Akhtar, N., \& Enns, J. T. (1989). Relations between covert orienting and filtering in the development visual attention. Journal of Experimental Child Psychology, 48, 315-334.

Aston-Jones, G., \& Cohen, J. D. (2005). An integrative theory of locus coeruleus-norepinephrine function: Adaptive gain and optimal performance. Annual Review of Neuroscience, 28, 403-450. doi:10.1146/ annurev.neuro.28.061604.135709

Aston-Jones, G., Rajkowski, J., \& Cohen, J. (1999). Role of locus coeruleus in attention and behavioral flexibility. Biological Psychiatry, 46, 1309-1320. doi:10.1016/S0006-3223(99)00140-7 
Berger, A., Jones, L., Rothbart, M. K., \& Posner, M. I. (2000). Computerized games to study the development of attention in childhood. Behavior Research Methods, Instruments, and Computers, 32, 297-303.

Bledowski, C., Prvulovic, D., Goebel, R., Zanella, F. E., \& Linden, D. E. J. (2004). Attentional systems in target and distractor processing: A combined ERP and fMRI study. NeuroImage, 22, 530-540. doi:10.1016/j .neuroimage.2003.12.034

Brodeur, D. A., \& Enns, J. T. (1997). Covert visual orienting across the lifespan. Canadian Journal of Experimental Psychology/Revue canadienne de psychologie expérimentale, 51, 20-35. doi:10.1037/11961961.51.1.20

Bush, G., Luu, P., \& Posner, M. I. (2000). Cognitive and emotional influences in anterior cingulate cortex. Trends in Cognitive Sciences, 4, 215-222. doi:10.1016/S1364-6613(00)01483-2

Callejas, A., Lupiáñez, J., Funes, M. J., \& Tudela, P. (2005). Modulations among the alerting, orienting and executive control networks. Experimental Brain Research, 167, 27-37. doi:10.1007/s00221-005-2365-Z

Callejas, A., Lupiáñez, J., \& Tudela, P. (2004). The three attentional networks: On their independence and interactions. Brain and Cognition, 54, 225-227. doi:10.1016/j.bandc.2004.02.012

Corbetta, M., \& Shulman, G. L. (2002). Control of goal-directed and stimulus-driven attention in the brain. Nature Reviews Neuroscience, 3, 201-215. doi:10.1038/nrn755

Davidson, M. C., Amso, D., Anderson, L. C., \& Diamond, A. (2006). Development of cognitive control and executive functions from 4 to 13 years: Evidence from manipulations of memory, inhibition, and task switching. Neuropsychologia, 44, 2037-2078. doi:10.1016/j.neuropsychologia.2006.02.006

Diamond, A. (2013). Executive functions. Annual Review of Psychology, 64, 135-168. doi:10.1146/annurev-psych-113011-143750

Dosenbach, N. U. F., Fair, D. A., Cohen, A. L., Schlaggar, B. L., \& Petersen, S. E. (2008). A dual networks architecture of top-down control. Trends in Cognitive Sciences, 12, 99-105. doi:10.1016/j.tics.2008.01 .001

Dye, M. W. G., Baril, D., \& Bavelier, D. (2007). Which aspects of visual attention does deafness modify? The case of the attentional network test. Neuropsychologia, 45, 1801-1811. doi:10.1016/j.neuropsychologia 2006.12 .019

Enns, J. T. (1990). Relations among components of visual attention. In J. T. Enns (Ed.), The development of attention: Research and theory (pp. 139-158). Amsterdam, the Netherlands: Elsevier.

Enns, J. T., \& Brodeur, D. A. (1989). A developmental study of covert orienting to peripheral visual cues. Journal of Experimental Child Psychology, 48, 171-189.

E-Prime (Version 2) [Computer software]. Sharpsburg, PA: Psychology Software Tools.

Fair, A. D., Dosenbach, N. U. F., Chusch, J. A., Cohen, A. L., Brahmbhatt, S., Miezin, F. M., . . Schlaggar, B. L. (2007). Development of distinct control networks through segregation and integration. Proceedings of the National Academy of Science of the United States of America, 104, 13507-13512. doi:10.1073/pnas.0705843104

Fan, J., McCandliss, B. D., Sommer, T., Ras, M., \& Posner, M. I. (2002). Testing the efficiency and independence of attentional networks. Journal of Cognitive Neuroscience, 14, 340-347. doi: 10.1162/089892902317361886

Fjell, A. M., Walhovd, K. B., Brown, T. T., Kuperman, J. M., Chung, Y., Hagler, D. J., Jr., . . Dale, A. M. (2012). Multimodal imaging of the self-regulating developing brain. Proceedings of the National Academy of Science of the United States of America, 109, 19620-19625. doi: 10.1073/pnas.1208243109

Fuentes, L. J., \& Campoy, G. (2008). The time course of alerting effect over orienting in the attention network test. Experimental Brain Research, 185, 667-672. doi:10.1007/s00221-007-1193-8
Gerardi-Caulton, G. (2000). Sensitivity to spatial conflict and the development of self-regulation in children 24-36 months of age. Developmental Science, 3, 397-404. doi:10.1111/1467-7687.00134

Houwink, A., Aarts, P. B. M., Geurts, A. C. H., \& Steenbergen, B. (2011). A neurocognitive perspective on developmental disregard in children with hemiplegic cerebral palsy. Research in Developmental Disabilities, 32, 2157-2163. doi:10.1016/j.ridd.2011.07.012

Johnson, K. A., Robertson, I. H., Barry, E., Mulligan, A., Dáibhis, A., Daly, M., . . Bellgrove, M. A. (2008). Impaired conflict resolution and alerting in children with ADHD: Evidence from the attention network task (ANT). Journal of Child Psychology and Psychiatry, 49, 1339 1347. doi:10.1111/j.1469-7610.2008.01936.x

Kaufman, A. S., \& Kaufman, N. L. (1990). Kaufman Brief Intelligence Test-Manual. Circle Pines, MN: American Guidance Service.

Konrad, K., Neufang, S., Thief, C. M., Specht, K., Hanisch, C., Fan, J., . . . Fink, G. R. (2005). Development of attentional networks: An fMRI study with children and adults. NeuroImage, 28, 429-439. doi:10.1016/ j.neuroimage.2005.06.065

Kratz, O., Studer, P., Malcherek, S., Erbe, K., Moll, G. H., \& Heinrich, H. (2011). Attentional processes in children with ADHD: An event-related potential study using the attention network test. International Journal of Psychophysiology, 81, 82-90. doi:10.1016/j.ijpsycho.2011.05.008

Kraut, A. G. (1976). Effects of familiarization on alertness and encoding in children. Developmental Psychology, 12, 491-496. doi:10.1037/00121649.12.6.491

Kray, J., Eber, J., \& Lindenberger, U. (2004). Age differences in executive functioning across the lifespan: The role of verbalization in task preparation. Acta Psychologica, 115, 143-165. doi:10.1016/j.actpsy.2003.12 .001

Mezzacappa, E. (2004). Alerting, orienting, and executive attention: Developmental properties and sociodemographic correlates in an epidemiological sample of young, urban children. Child Development, 75, 1373 1386. doi:10.1111/j.1467-8624.2004.00746.x

Morrison, F. J. (1982). The development of alertness. Journal of Experimental Child Psychology, 34, 187-199. doi:10.1016/00220965(82)90041-8

Ortega, R., López, V., Carrasco, X., Anllo-Vento, L., \& Aboibitz, F. (2013). Exogenous orienting of visual-spatial attention in ADHD children. Brain Research, 1493, 68-79. doi:10.1016/j.brainres.2012.11.036

Pearson, D. A., \& Lane, D. M. (1991). Auditory attention switching: A developmental study. Journal of Experimental Child Psychology, 51, 320-334. doi:10.1016/0022-0965(91)90039-U

Petersen, S. E., \& Posner, M. I. (2012). The attention system of the human brain: 20 years after. Annual Review of Neuroscience, 35, 73-89. doi:10.1146/annurev-neuro-062111-150525

Posner, M. I. (1978). Chronometric exploration of mind. Hillsdale, NJ: Erlbaum.

Posner, M. I. (1980). Orienting of attention. Quarterly Journal of Experimental Psychology, 32, 3-25.

Posner, M. I. (1994). Attention: The mechanism of consciousness. Proceedings of the National Academy of Science of the United States of America, 91, 7398-7403. doi:10.1073/pnas.91.16.7398

Posner, M. I., Rothbart, M. K., Sheese, B. E., \& Tang, Y. (2007). The anterior cingulate gyrus and the mechanism of self-regulation. Cognitive, Affective, \& Behavioral Neuroscience, 7, 391-395. doi:10.3758/ CABN.7.4.391

Posner, M. I., Rueda, M. R., \& Kanske, P. (2007). Handbook of psychophysiology: Probing the mechanisms of attention (pp. 410-432). Cambridge, England: Cambridge University Press. doi:10.1017/ CBO9780511546396.018

Quintero-Gallego, E. A., Gómez, C. M., Morales, M., \& Márquez, J. (2011). Spatial orientation deficit in children due to cerebellum astrocytoma pediatric tumor obtained by means of the attentional network 
test. Neuroscience Letters, 504, 232-236. doi:10.1016/j.neulet.2011.09 .034

Ridderinkhof, K. R., Ullsperger, M., Crone, E. A., \& Nieuwenhuis, S. (2004, October 15). The role of the medial frontal cortex in cognitive control. Science, 306, 443-447. doi:10.1126/science.1100301

Ridderinkhof, K. R., \& van der Molen, M. W. (1995). A psychophysiological analysis of developmental differences in the ability to resist interference. Child Development, 66, 1040-1056. doi:10.2307/1131797

Ridderinkhof, K. R., van der Molen, M. W., Band, G. P. H., \& Bashore, T. R. (1997). Sources of interference from irrelevant information: A developmental study. Journal of Experimental Child Psychology, 65, 315-341. doi:10.1006/jecp.1997.2367

Rueda, M. R. (2014). Development of attention. In K. Ochsner \& S. Kosslyn (Eds.), Oxford handbook of cognitive neuroscience (pp. 296318). New York, NY: Oxford University Press. doi:10.1093/oxfordhb/ 9780199988693.013.0015

Rueda, M. R., Checa, P., \& Cómbita, L. M. (2012). Enhanced efficiency of the executive attention network after training in preschool children: Immediate changes and effects after two months. Developmental Cognitive Neuroscience, 2(Suppl. 1), 192-204. doi:10.1016/j.den.2011.09 .004

Rueda, M. R., Fan, J., McCandliss, B. D., Halparin, J. D., Gruber, D. B., Lercari, L. P., \& Posner, M. I. (2004). Development of attentional networks in childhood. Neuropsychologia, 42, 1029-1040. doi:10.1016/ j.neuropsychologia.2003.12.012

Rueda, M. R., Posner, M. I., \& Rothbart, M. K. (2005). The development of executive attention: contributions to the emergence of selfregulation. Developmental Neuropsychology, 28, 573-594. doi: $10.1207 / \mathrm{s} 15326942 \mathrm{dn} 2802 \_2$

Schul, R., Townsend, J., \& Stiles, J. (2003). The development of attentional orienting during the school-age years. Developmental Science, 6, 262272. doi:10.1111/1467-7687.00282
Smothergill, D. W., \& Kraut, A. G. (1989). Developmental studies of alertness and encoding effects of stimulus repetition. Advances in Child Development and Behavior, 22, 249-270. doi:10.1016/S00652407(08)60416-4

Tao, L., Marzecová A., Taft, M., Asanowicz, D., \& Wodniecka, Z. (2011). The efficiency of attentional networks in early and late bilinguals: The role of age of acquisition. Frontiers in Psychology: Cognition, 2, 123. doi: $10.3389 /$ fpsyg.2011.00123

Trick, L. M., \& Enns, J. T. (1998). Lifespan changes in attention: The visual search task. Cognitive Development, 13, 369-386. doi:10.1016/ S0885-2014(98)90016-8

Wainwright, A., \& Bryson, S. E. (2002). The development of exogenous orienting: Mechanisms of control. Journal of Experimental Child Psychology, 82, 141-155. doi:10.1016/S0022-0965(02)00002-4

Waszak, F., Li, S. C., \& Hommel, B. (2010). The development of attentional networks: Cross-sectional findings from a life span sample. $D e-$ velopmental Psychology, 46, 337-349. doi:10.1037/a0018541

Weinbach, N., \& Henik, A. (2012). The relationship between alertness and executive control. Journal of Experimental Psychology: Human Perception and Performance, 38, 1530-1540. doi:10.1037/a0027875

Yang, S., Yang, H., \& Lust, B. (2011). Early childhood bilingualism leads to advances in executive attention: Dissociating culture and language. Bilingualism: Language and Cognition, 14, 412-422. doi:10.1017/ S1366728910000611

Yerkes, R. M., \& Dodson, J. D. (1908). The relation of strength of stimulus to rapidity of habit-formation. Journal of Comparative Neurology \& Psychology, 18, 459-482. doi:10.1002/cne.920180503

Received July 11, 2013

Revision received May 16, 2014

Accepted June 18, 2014 